ORIENTAL JOURNAL OF CHEMISTRY

An International Open Free Access, Peer Reviewed Research Journal

www.orientjchem.org
ISSN: 0970-020 X

CODEN: OJCHEG

2014, Vol. 30, No. (2):

Pg. 833-835

\title{
An Efficient an One-Pot Method for Synthesis of 2-Aryl-(3H)-4-Quinazolinones Derivatives Catalyzed by SSA
}

\author{
FARHAD HATAMJAFARI* and SIMA ESLAMI \\ Department of Chemistry, Faculty of Science, \\ Islamic Azad University-Tonekabon Branch, Tonekabon, Iran. \\ ${ }^{*}$ Corresponding author E-mail: hatamjafari@yahoo.com \\ http://dx.doi.org/10.13005/ojc/300259
}

(Received: February 02, 2014; Accepted: March 04, 2014)

\begin{abstract}
A mixture of 2-amino benzamide with aromatic aldehyds in the presence of SSA under solvent-free condition were converted to quinazolinones with good yields.
\end{abstract}

Key words: Starch SulphuricAcid (SSA), (3H)-4-Quinazolinones, One-pot, Solvent-Free .

\section{INTRODUCTION}

Natural compounds with quinazoline structural motif have been identified to display a wide range of biological activities. The prominent potencies associated with quinazoline containing compounds are their anticancer,anti-inflammatory, anthelmintic, analgesic, and anticonvulsive activities $^{1-5}$.

We now report that cyclocondensation of 2-aminobenzamides (1) with aromatic aldehyds(2) on silica-supported sulfuric acid quickly provides the substituted quinazolin-4(3H)-ones (3) in fairly high yields. To our knowledge there has been reported on the use of sulfuric acid adsorbed on silica gel $\left(\mathrm{SiO}_{2} \mathrm{H}_{2} \mathrm{SO}_{4}\right)$ as a solid catalyst in the synthesis of quinazolines. Various catalysts were used in the synthesis of quinazolinone derivatives ${ }^{6-}$ 14.

The early method, based on the reaction of 2-aminobenzamide with aldehyds, was performed in the presence of $\mathrm{H}_{2} \mathrm{SO}_{4}$ and as a requirement of its refluxing condition much excess of aldehyds was used ${ }^{15}$.Though an excess of aldehyds is necessary to diminish the intermolecular side-reactions, the conventional beating method ever needs additional aldehyds both to improve the yield. In contrast to the Liquid $\mathrm{H}_{2} \mathrm{SO}_{4}$, which is difficult to recovery and takes some effort in workup, the silica-supported $\mathrm{H}_{2} \mathrm{SO}_{4}$, can be easily separated and recycled without the loss of activity for several times. Thus, 2-aminobenzamides (1) reacted with nearly 2 equivalents of aromatic aldehyds(2) with the aide of $\mathrm{SiO}_{2,} \mathrm{H}_{2} \mathrm{SO}_{4}$ and 
submission to microwave irradiation to provide fairly high yields of substituted quinazolin-4(3H)-ones (2a-d) in 25 minutes.

However, these reactions often require harsh conditions and long reaction time and low efficiency can. Aromatic aldehydes, especially when used to replace for synthesis quinazolinones. Previously, we have synthesized a number of heterocyclic compounds ${ }^{16-28}$. Herein we report some of quinazolinones at one pot reaction, environmentally friendly with high yields and easy separation (Scheme 1).<smiles>NC(=O)c1ccccc1N</smiles><smiles>[I-][I-]</smiles><smiles>[X]c1ccc(C=O)cc1</smiles><smiles>CC(C)O[Si]</smiles><smiles>O=c1[nH]c(Br)nc2ccccc12</smiles>

Scheme 1:

General Procedure for the Preparation of (3H)-4Quinazolinone Derivatives

A mixture of aldehyde $(1 \mathrm{mmol})$ and2amino benzamide $(1 \mathrm{mmol})$, and starch sulphuric acid $(20 \mathrm{~W} \%)$ as a catalyst was stirred at $80^{\circ} \mathrm{C}$ for $25 \mathrm{~min}$. The progress of reaction was monitored by TLC. After finishing, recrystallized from ethanol $95 \%$ to give pure products $(2 \mathrm{a}-\mathrm{d})$

\section{2-(3-nitrophenyl)-(3H)-4-Quinazolinone (2a)}

Pale Blue crystals, Yield: 87\%, m.p> 300 ${ }^{\circ} \mathrm{C}, \mathrm{FT}-\mathrm{IR}\left(\mathrm{V}_{\text {max }} / \mathrm{cm}^{-1}\right)$ (KBr disc): 3440 (amid NH Str.); 3092(arom CH Str.); 1682(amid C=O Str.); 1640(C=N Str.); 1520, $1360(\mathrm{~N}-\mathrm{O}$ Str. $) .{ }^{1} \mathrm{H}$ $\operatorname{NMR}(400.13 \mathrm{MHz}, \mathrm{DMSO}): \delta=7.56\left(1 \mathrm{H}, \mathrm{t},{ }^{3} \mathrm{~J}=8 \mathrm{~Hz}\right.$, $1 \mathrm{CH})$; 7.79-7.88 $(3 \mathrm{H}, \mathrm{m}, 3 \mathrm{CH}) ; 8.17\left(1 \mathrm{H}, \mathrm{d},{ }^{3} \mathrm{~J}=8 \mathrm{~Hz}\right.$, $1 \mathrm{CH}) ; 8.42\left(1 \mathrm{H}, \mathrm{d},{ }^{3} \mathrm{~J}=8 \mathrm{~Hz}, 1 \mathrm{CH}\right) ; 8.61\left(1 \mathrm{H}, \mathrm{d},{ }^{3} \mathrm{~J}=\right.$ $8 \mathrm{~Hz}, 1 \mathrm{CH}) ; 9.02(1 \mathrm{H}, \mathrm{s}, 1 \mathrm{CH}) ; 11.82(1 \mathrm{H}, \mathrm{s}, \mathrm{NH})$.

\section{2-(4-methoxyphenyl)-(3H)-4-Quinazolinone (2b)}

Yellow crystals, Yield: \%72, m. $\mathrm{p}=245-248$ ${ }^{\circ} \mathrm{C}$, FT-IR $\left(\mathrm{V}_{\max } / \mathrm{cm}^{-1}\right)(\mathrm{KBr}$ disc): 3410 (amid NH Str.); 3131(arom. CH Str.); 2990(aliph. CH Str.); 1675(amid C=O Str.); 1643 (C=N Str.).

\section{2-(4-chlorophenyl)-(3H)-4-Quinazolinone (2c)}

White crystals, Yield: $89 \%$, m. $p=302-304$
${ }^{\circ} \mathrm{C}$, FT-IR $\left(\mathrm{V}_{\mathrm{max}} / \mathrm{cm}^{-1}\right)$ ( $\mathrm{KBr}$ disc): 3410 (amid .NH str.); 3078 (arom. CH str.); 1670(amid. C=O Str.); 1648(C=N Str.). ${ }^{1} \mathrm{H}$ NMR ( $\left.400.13 \mathrm{MHz}, \mathrm{DMSO}\right)$ : $\delta=7.53\left(1 \mathrm{H}, \mathrm{t},{ }^{3} \mathrm{~J}=7.8,1 \mathrm{CH}\right) ; 7.63\left(2 \mathrm{H}, \mathrm{d},{ }^{3} \mathrm{~J}=7.8,2 \mathrm{CH}\right)$; $7.75\left(1 \mathrm{H}, \mathrm{d},{ }^{3} \mathrm{~J}=7.81 \mathrm{CH}\right) ; 7.84\left(1 \mathrm{H}, \mathrm{t},{ }^{3} \mathrm{~J}=7.8,1 \mathrm{CH}\right)$; $8.16\left(1 \mathrm{H}, \mathrm{d},{ }^{3} \mathrm{~J}=7.8,1 \mathrm{CH}\right) ; 8.21\left(2 \mathrm{H}, \mathrm{d},{ }^{3} \mathrm{~J}=7.8,2 \mathrm{CH}\right)$; $11.54(1 \mathrm{H}, \mathrm{s}, \mathrm{NH})$.

\section{2-(4-nitrophenyl)-(3H)-4-Quinazolinone (2d)}

Pale yellow crystals, Yield: $69 \%, \mathrm{~m} . \mathrm{p}=361$ $363^{\circ} \mathrm{C}$,FT-IR $\left(\mathrm{V}_{\mathrm{max}} / \mathrm{cm}^{-1}\right)(\mathrm{KBr}$ disc): 3380 (amid. $\mathrm{NH}$ Str.); 3050(arom. CH Str); 1678(amid. C=O Str.); 1635(C=N Str.); 1530, 1355(N-O Str.).H NMR ( $400.13 \mathrm{MHz}$, DMSO): $\delta=7.56\left(1 \mathrm{H}, \mathrm{t},{ }^{3} \mathrm{~J}=7.2,1 \mathrm{CH}\right)$; $7.78\left(1 \mathrm{H}, \mathrm{d},{ }^{3} \mathrm{~J}=7.2,1 \mathrm{CH}\right) ; 7.86\left(1 \mathrm{H}, \mathrm{t},{ }^{3} \mathrm{~J}=7.2,1 \mathrm{CH}\right)$; $8.17\left(1 \mathrm{H}, \mathrm{d},{ }^{3} \mathrm{~J}=7.2, \mathrm{CH}\right) ; 8.37-8.42\left(4 \mathrm{H}, \mathrm{dd},{ }^{3} \mathrm{~J}=8.8\right.$, $\mathrm{CH}) ; 11.41(1 \mathrm{H}, \mathrm{s}, \mathrm{NH})$.

In conclusion, in comparison with the precedent related methods which show drawbacks from the standpoints of, prolong refluxing in solvent, need to inert gas, and consuming more aldehyds, the notable advantages of this methodology are: mild conditions in a solvent-free system, fairly high yields, fast reaction, no aqueous work-up and safe and environmental friendly conditions.

\section{REFERENCES}

1. Gellibert F., Fouchet M. H., Nguyen V.L., Wang R., Krysa G., de Gouville A. C., Huet S. and Dodic N. Bioorg. Med. Chem. Lett., 2009. 19:

\section{7}

2. Khan M.T. H., Khan R., Wuxiuer Y., Arfan M., Ahmed M. and Sylte I. Bioorg. Med. Chem. 
2010., 18: 4317

3. Azizian J., Shameli A., Balalaie S., Ghanbari M. M., Zomorodbakhsh S., Entezari M., Bagheri S. and Fakhrpour G. Orient. J. Chem. 2012, 28: 327.

4. Mulakayala N., Kandagatla B., Ismail Rapolu R. K., Rao P., Mulakayala C., Kumar C. S., Iqbal J. and Oruganti S. Bioorg. Med. Chem. Lett. 2012., 22: 5063

5. Adib M., Ansari S., Mohammadi A. and Bijanzadeh H. R. Tetrahedron Lett. 2010., 51: 30

6. Zhou J. and Fang J. J. Org. Chem. 2011., 76: 7730

7. Roopan S. M., Maiyalagan T. and Khan F. N. Can. J. Chem. 2008., 86: 1019

8. Tavakoli-Hoseini N. and Davoodnia A. Chin. J. Chem. 2011., 29: 1685

9. Wang X-S., Yang K., Zhang M-M. and Yao CS. Synth. Commun. 2010., 40: 2633

10. Chen J., Wu D., He F., Liu M., Wu H., Ding J. and Su W. Tetrahedron Lett. 2008., 49: 3814

11. Tavakoli-Hoseini N. and Davoodnia A. Synth. React. Inorg. Met. Org. Chem. 2012., 42: 76

12. MohammadiZiarani G., Lashgari N. and Badiei A. Sci. Iran. 2013., 20: 580

13. MohammadiZiarani G., Badiei A., Mousavi M., Lashgari N. and Shahbazi A. Chin. J. Catal. 2012., 33:1832

14. MohammadiZiarani G., Badiei A., Dashtianeh Z., Gholamzadeh P. and HosseiniMohtasham N. Res. Chem.
Intermed. 2013, 39: 3157.

15. Mckee M K., Mckee R L. and Bost RW. J. Am. Chem. Soc. 1947., 69: 184

16. Azizian J., Hatamjafari F., Karimi A. R. and Shaabanzadeh M., Synthesis, 2006., 5: 765

17. Azizian J., Shaabanzadeh M., Hatamjafari F. and Mohammadizadeh M.R., Arkivoc, 2006. $x i: 47$

18. Hatamjafari F., Synthetic Communications, 2006. ý36: 3563

19. Azizian J., Hatamjafari F. and Karimi A. R., Journal of Heterocyclic Chemistry,, 2006.43:1349

20. Hatamjafari F and Montazeri N., Turkish Journal of Chemistryý,2009.33: 797

21. Bidram A., Hatamjafari $F$ and Doryeh A., Orient. J. Chem. 2013., 29: 123

22. Hatamjafari F., Orient. J. Chem. 2012., 28: 141

23. Hatamjafari F., Orient. J. Chem. 2013., 29: 93

24. Hatamjafari $F$ and Alijanichakoli F., Orient. J.Chem. 2013.,29: 145

25. Hatamjafari $F$ and Hosseinian A., Orient. J.Chem. 2013.,29: 109

26. Hatamjafari F and Keyhani A., Orient. J.Chem. 2013,29: 783.

27. Hatamjafari F and Khojastehkouhi H., Orient. J. Chem, 2014, 301, 329-331.

28. Hatamjafari F and GermaniNezhad F., Orient. J. Chem., 2014, 301, 355-357. 